\title{
Inhibition of Coxsackievirus A24 in permissive Hep2 cells by small interfering RNA (siRNA)
}

\author{
Abhishek Kumar Mishra, Gita Satpathy \\ (Department of Microbiology /All India Institute of Medical Sciences, New Delhi, India)
}

\begin{abstract}
RNA interference is a sequence specific post transcriptional gene silencing mechanism which works through cleaving of nucleic acids by small RNA molecules of 19-21 mers. RNA interference tool found very effective to destroy the pathogenicity of several viruses. The molecular scissor activity of small interfering RNA (siRNA) was applied for inhibition of CoxsackievirusA24 (CA24), an Enterovirus of family Picornaviridae responsible for acute haemorrhagic conjunctivitis in humans. Four different siRNA molecules were used to target 5' untranslated region (si5U), cis acting replication element of $2 \mathrm{C}$ (siCre), RNA dependent RNA polymerase enzyme coding region (si3D ${ }^{\text {pol }}$ ) and 3' untranslated region of CA24. Virus inhibition study by siRNA was done in Hep2 cells. Cytopathic effect, immunofluorescence assay, 50\% tissue culture infectious dose

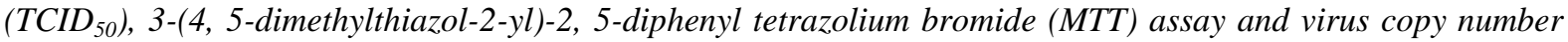
in Real-time PCR assays were used for the validation of virus inhibition potential of designed siRNA. Analysis of cumulative data reflected that si5U and si3D ${ }^{\text {pol }}$ are highly efficient to stop CA24 propagation in Hep2 cells. Transfected Hep2 cells with any of these siRNA found refrained for CA24 infection in cell culture, immunofluorescence assay, $T C I D_{50}$ and Real Time PCR assay ( $\left.p<0.05\right)$.
\end{abstract}

Keywords: Acute haemorrhagic conjunctivitis, cytopathic effect, Immunofluorescence, RNA interference

\section{Introduction}

CoxsackievirusA24 (CA24) is one of the major infectious virus of family Picornaviridae, responsible for acute hemorrhagic conjunctivitis (AHC)-a highly contagious infection of eye, characterized by rapid onset of painful red eye, conjunctival edema, congestion and sub-conjunctival hemorrhages [1]. In past few decades CA24 emerged as major pathogen responsible for several AHC outbreaks not only in India but other countries like, Spain, Brazil, China, Cuba, Taiwan [2, 3, 4, 5, 6, 7]. Attempts were made to develop anti-CA24 therapeutics, still, no effective medication is available to treat CA24 infections. RNAi tool found very effective against a wide range of viruses both in vitro and in vivo, including Coxsackievirus groups, Dengue virus, Ebola virus, Human Immunodeficiency virus, Hepatitis $C$ virus $[8,9,10,11,12]$. CA24 is a non-enveloped, positive sense single-stranded RNA virus of $\sim 7.5 \mathrm{~kb}$ in length. The genome of CA24 is arranged in 5' untranslated region (5'UTR), a single open reading frame (ORF) and 3'untranslated region (3'UTR) followed by a poly (A) tail. The four capsid proteins (VP1-4) and seven non-structural proteins (2A, 2B, 2C, 3A, 3B, 3C and 3D) are generated by proteolytic cleavage of a single polyprotein translated from the genomic RNA.

Single stranded positive sense RNA genome of CA24 works as both a replication template and messenger RNA. Therefore, nucleic acid based therapeutics may be very useful to destroy transcription and translation of CA24 i.e., complete destruction of virus $[13,14]$.Successful inhibition of CA24 by siRNA was done earlier targeting 3D and Cre regions $[15,16]$. In this study, instead of these regions, both of the untranslated regions (5'UTR and 3' UTR) of CA24 was also targeted.

\section{Materials And Methods}

2.1. Cells and virus: Human epidermoid cancer cells (Hep-2) was procured from National Centre for Cell Science (NCCS), Pune, India, and maintained in $25 \mathrm{~cm}^{2}$ cell culture flasks (Nunc, USA) at $37^{\circ} \mathrm{C}$ in a $5 \% \mathrm{CO}_{2}$ incubator (New Brunswick, USA). All cell culture experiments were performed in an aseptic condition within a class II Biosafety cabinet (Thermo EC, USA). Cells were sub-cultured at a split ratio of 1:3 after treatment with $0.25 \%$ Trypsin-EDTA solution [17] followed by addition of complete cell culture medium containing Dulbecco's Modified Eagle's Medium (Invitrogen) supplemented with 10\% heat inactivated fetal bovine serum (Invitrogen), 100 units penicillin/ml, 10 microgram streptomycin/ml and 25 microgram of amphotericin B/ml (Sigma- Aldrich). CA24 (ATCC-VR-583) was procured from the American Type Culture Collection (ATCC, USA). Tissue culture infection dose $\left(\mathrm{TCID}_{50}\right)$ method was used to determine the virus titer [18]. Briefly, Hep-2 cells was seeded in 24 well flat bottom plates (Corning, USA). Sub confluent monolayer of cells were washed thrice with serum free media and inoculated with $200 \mu$ l of virus (1:1000 dilutions from stock) and incubated for $2 \mathrm{~h}$ at $37^{\circ} \mathrm{C}$ in a $5 \% \mathrm{CO}_{2}$ incubator to allow virus adsorption. Inoculum was removed and $1 \mathrm{ml}$ of maintenance medium (cell culture medium containing 2\% FBS) was added in each well of the plate. Plate was incubated and observed regularly for the appearance of cytopathic effect under an inverted light microscope (Nikon, Japan). All titrations were done using each single dilution in four wells.

DOI: 10.9790/3008-1203042126 www.iosrjournals.org $\quad 21 \mid$ Page




\subsection{Designing and synthesis of small interfering RNAs (siRNA):}

To design small interfering RNA (siRNA), full genome of CA24 (Genbank accession no. D90457.1) was screened using siRNA target finder (Ambion, USA). Four siRNA was chosen against 5' untranslated region (si5U), cis acting replication element of $2 \mathrm{C}$ (si2C), RNA dependent RNA polymerase coding region (si3 $\mathrm{D}^{\mathrm{pol}}$ ) and 3' untranslated region (si3U) of CA24 (Table.1). All four siRNA were put on BLAST search to ensure specificity for viral regions only. Designed siRNA was synthesized by Sigma (Sigma Aldrich, USA).

\subsection{Antiviral potency of designed siRNA:}

To test anti-CA24 potential of designed siRNA, sub confluent monolayers of cells was individually transfected with 100 nanomolar (NM) of each siRNA using Lipofectamine ${ }^{\mathrm{TM}} 2000$ (Invitrogen, USA). Lipofectamine: siRNA was used in 1:1 ratio for this transfection. Twenty four hour post transfection, cells were inoculated with $100 \mathrm{TCID}_{50}$ of CA24. Propagation of CA24 in transfected cells were assessed in cytopathic effect (CPE), 50\% tissue culture infectious dose ( $\mathrm{TCID}_{50}$ ), 3-(4, 5-dimethylthiazol-2-yl)-2,5-diphenyltetrazolium bromide assay (MTT assay), immunofluorescence assay (IFA) and quantitative real-time RT-PCR (Q-RT PCR) assays. Non-treated Hep2 cells, CA24 infected Hep2 cells, mock transfected cells and scrambled siRNA transfected cells were used as controls in all assays. All experiments were performed in 3 biological and 3 technical replicates.

\subsubsection{CPE and TCID 50 determination:}

Cells transfected with siRNA, followed by inoculation with $100 \mathrm{TCID}_{50}$ of CA24 were observed periodically under the inverted microscope (Nikon, USA). TCID $_{50}$ was calculated 72 hours post-infection using Reed and Muench method [18].

\subsubsection{Indirect immunofluorescence assay (IFA):}

Hep2 cells were seeded over $22 \mathrm{~mm}$ round cover slips (Blue star, India) placed in 12 well tissue culture plates (Corning, USA) and incubated to reach $80 \%$ confluency at the time of transfection. Cells were transfected and infected in the same manner as described before in this paper and incubated for 72 hours. Coverslips were washed thrice with phosphate buffer saline (PBS, pH 7.4) and cells were fixed using chilled methanol. Fixed cells were treated with mouse monoclonal antibody at 1:30 dilution against CA24 (Chemicon, USA). Plates were incubated at $37^{\circ} \mathrm{C}$ for 1 hour in a dark humidified chamber, followed by washing thrice in a sequential manner with PBS and distilled water. Cells were treated with FITC conjugated rabbit anti-mouse Ig-G at 1:500 dilutions (Dako, Denmark) for 30 minutes, followed by washing. Cells were mounted and observed under a fluorescence microscope (Nikon, Japan).

\subsubsection{Real Time PCR (RT PCR):}

Total RNA was extracted from CA24 infected cells and reverse transcribed using Superscript III RT (Invitrogen) and reverse primers specific for partial regions of- 5'UTR (337-536), Cre (4550-4742), 3D ${ }^{\text {pol }}$ (6334-6533) and 3'UTR (7281-7461). The product of RT PCR was amplified using Pfu DNA polymerase (Promega) and cloned into pPCRScript SK+ cloning vector (Stratagene, USA) as per the manufacturer's instructions. Glyceraldehyde-3-phosphate dehydrogenase (GAPDH) was used for normalization of data. Log dilution of the cloned fragments was prepared in the range of $10^{-2}$ to $10^{-14}$ and used to generate the standard plot. Total RNA was extracted from transfected and CA24 infected cells and equal quantity of RNA was reverse transcribed. Real-Time PCR carried out in a real-time PCR machine (CFX-96, Bio-Rad) using a microseal 96 well-skirted PCR plate (MSP-9601). The reaction mixture was prepared in $20 \mu$ l volume containing $10 \mu \mathrm{l}$ of Sso Fast Eva Green supermix (Bio-Rad, USA), $5.6 \mu \mathrm{l}$ of nuclease free water, $0.8 \mu \mathrm{l}(10 \mu \mathrm{M})$ of each sense and antisense primer and $1 \mu \mathrm{l}$ of template. The thermal profile used for amplification- an initial denaturation at $95^{\circ} \mathrm{C}$ for 2 minutes, followed by 40 cycles of denaturation $\left(95^{\circ} \mathrm{C}\right.$ for $\left.1 \mathrm{~min}\right)$, annealing 1 minute, extension at $72^{\circ} \mathrm{C}$ for 1 minute and final extension at $72^{\circ} \mathrm{C}$ for 7 minutes. Annealing temperature was $51^{\circ} \mathrm{C}$ for 5 ' $\mathrm{UTR}, 50^{\circ} \mathrm{C}$ for $\mathrm{Cre}$ and $3 \mathrm{D}^{\mathrm{pol}}, 56^{\circ} \mathrm{C}$ for $3^{\prime} \mathrm{UTR}$ and $45^{\circ} \mathrm{C}$ for GAPDH. A melting curve was also analyzed within the temperature range of $55^{\circ} \mathrm{C}$ to $95^{\circ} \mathrm{C}$. To avoid any wrong interpretation, residual nucleic acids were destroyed and removed by three successive nuclease (DNAse / RNAse) treatments.

\subsection{Statistical analysis:}

All experiments were carried out in three independent biological and technical replicates. The cumulative values are expressed as a mean \pm standard deviation. Student's $t$ test was applied for data analysis and a p-value less than or equal to 0.05 was considered as significant. 


\subsection{Low or no infection in siRNA transfected cells:}

\section{Results}

Prominent CPE were seen in infection control, mock and scrambled siRNA transfected cells in form of cell rounding, clumping, syncytium formation, nuclear condensation and granulation inside the cytoplasm (Fig.1B-D).Seventy two hour post infection, cytopathic effect was $1.67 \pm 0.47 \%, 11.67 \pm 2.36 \%, 2 \pm 0.8 \%$ and $113.33 \pm 4.71 \%$ for si5U, siCre, si3D and si3U transfected cells respectively. In non-infected Hep2 cells few dead cells were seen as a result of over confluence.

\subsection{Virus titer $\left(\mathrm{TCID}_{50}\right)$ assay:}

The TCID50 / $\mathrm{ml}$ was determined at the end of 72 hours of CA24 infection and found $10^{2.46}$ for si5U, $10^{4.41}$ for siCre, $10^{2.8}$ for si3 $\mathrm{D}^{\mathrm{pol}}$ and $10^{4.59}$ for si3U treated Hep2 cells (Fig.3). No significant difference was observed in $\mathrm{TCID}_{50} / \mathrm{ml}$ of infection control $\left(10^{7.68}\right)$ and mock group $\left(10^{7.44}\right)$.

\subsection{Reduction of fluorescence in CA24 infected Hep2 cells:}

Seventy two hour post infection, level of fluorescence in si5U, siCre, si3Dpol and si3U transfected cells was $3.67 \pm 0.94 \%, 23.33 \pm 4.71 \%, 3 \pm 0.81 \%$ and $21.67 \pm 2.35 \%$ respectively. No fluorescence was seen in non-treated cells (Fig.2A) whereas prominent apple green fluorescence was seen in infection control, mock and scrambled siRNA transfected cells (Fig.2B-D).

\subsection{Inhibition of virus replication/copy number in Real-Time PCR assay:}

Seventy two hour post infection, virus copy number was $1 \pm 0.47 \%, 11.67 \pm 6.23 \%, 1.67 \pm 0.47 \%$ and $13.33 \pm 4.71 \%$ was found in 100 nanomolar of si5U, siCre, si3 $\mathrm{D}^{\text {pol }}$ and si3U transfected cells respectively (Fig.3).

\section{Figures And Tables}

Table - 1: Nucleotide position and sequence of designed siRNA:

\begin{tabular}{|c|c|c|c|}
\hline S.N & siRNAs & Sequences (Sense) 5' to 3' & Position \\
\hline 1. & 5'UTR $(\mathrm{si5U})$ & GAGUCUAUUGAGCUACUUA & $427-445$ \\
\hline 2. & $2 \mathrm{C}(\mathrm{siCre})$ & TCCATTGCCACATCATTAA & $4552-4570$ \\
\hline 3. & RDRP $\left(\mathrm{si3} \mathrm{D}^{\mathrm{pol}}\right)$ & GAGAGAUAUAUCCUGAACA & $6390-6408$ \\
\hline 4. & 3'UTR $\left.(\mathrm{si3})^{\prime}\right)$ & UUGGAUUAUUGGGUUAUAC & $7415-7433$ \\
\hline
\end{tabular}

Fig.1. Inhibition of cytopathic effect of CA24 in siRNA transfected Hep2 cells:



$\mathrm{E}$

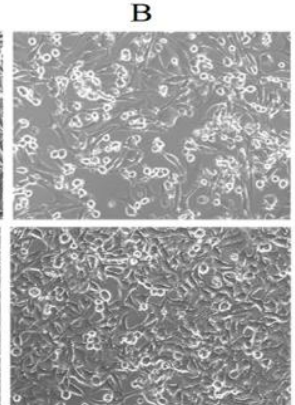

F

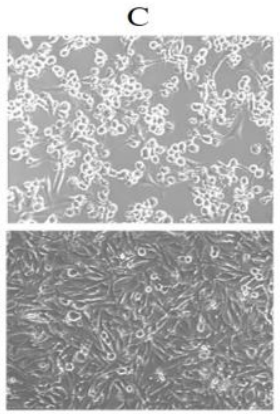

G



H

Fig.1. Inverted light microscopic images of Hep2 cells at 20X showing (A) Healthy Hep2 cells (B) CA24 infected Hep2 cells (C) Mock transfected Hep2 cells (D)-(H) Hep2 cells transfected with 100 nanomolar of scrambled siRNA, si5U, siCre, si3 $\mathrm{D}^{\text {pol }}$ and si3U respectively.

Fig.2. Inhibition of CA24 generated immunofluorescence in siRNA transfected Hep2 cells:


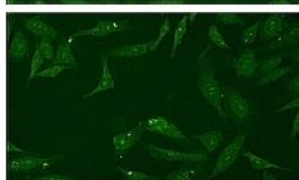

$\mathrm{E}$

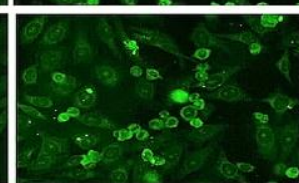

$\mathrm{F}$

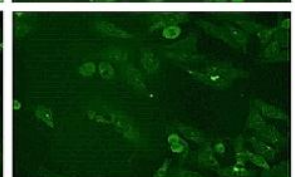

G



H

Fig.2. Immunofluorescence microscopic images of Hep2 cells at $20 \mathrm{X}$ showing (A) Healthy Hep2 cells (B) CA24 infected Hep2 cells (C) Mock transfected Hep2 cells (D)-(H) Hep2 cells transfected with 100 nanomolar of scrambled siRNA, si5U, siCre, si3 $\mathrm{D}^{\text {pol }}$ and si3U respectively. 
Fig.3. Cytoprotective and CA24 inhibition potential of pre transfected siRNA in Hep2 cells:

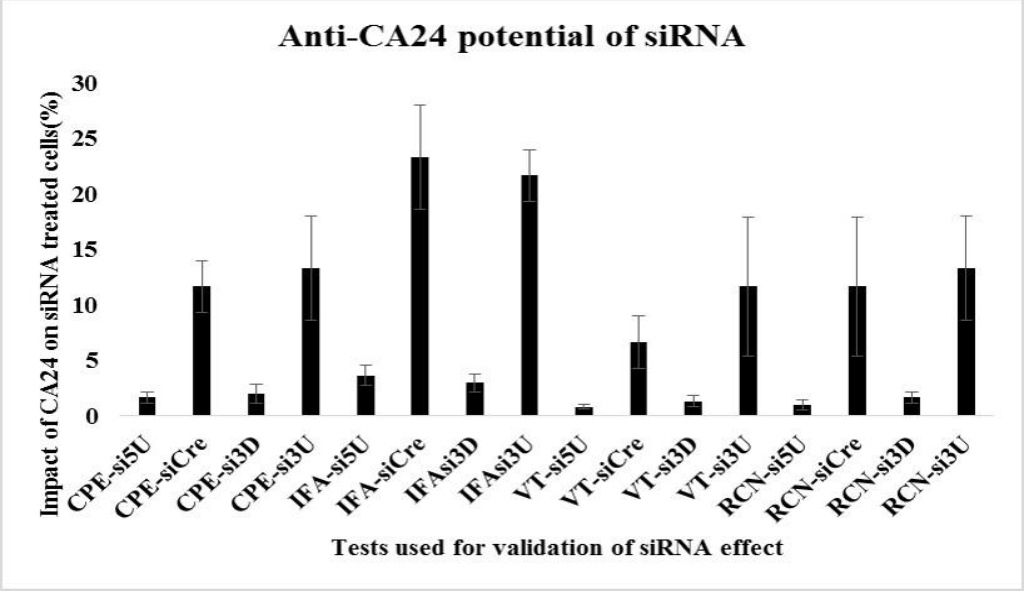

The graph showing inhibition of CA24 in Hep2 cells. CPE, IFA, TCID 50 and Real Time PCR data shown anti$\mathrm{CA} 24$ potential of si5U and si3 ${ }^{\mathrm{pol}}$. Data represented here as Mean $\pm \mathrm{SD}$.

\section{Discussion}

AHC was recognized as a major infection of eyes world over due to its short incubation time and high secondary attack rate with an explosive onset of signs and symptoms. The disease was noted in 1969 in Ghana, West Africa, from where it spread as a pandemic to the rest of the world [19].

In this study, we investigated the maximum possible inhibition of CA24 in Hep2 cells with our designed siRNA. Both of the untranslated regions (5'UTR and 3'UTR), Polymerase ( $\left.3 \mathrm{D}^{\mathrm{pol}}\right)$ and Cre of $2 \mathrm{C}$ were chosen as RNAi targets as these regions are essential for the survival of virus and their destruction could inhibit the virus propagation. In CA24, 5' UTR play a key role in replication and translation. Its high degree of conservation makes it less likely to mutate and the region of functional importance for viral replication. Therefore, this region is an attractive target for RNAi-based therapeutics. Earlier, siRNA against this region were successful in inhibition of plenty of viruses including Hepatitis $\mathrm{C}$ virus, Human immunodeficiency virus and CoxsackievirusB3 [20,21,22].The high order structure of 5'UTR makes targeting of this region especially challenging. However, in case of HCV, highly structured internal ribosome entry site (IRES) of 5'UTR was used as the most successful RNAi target [23]. As major components of virus propagation lies in 5'UTR of CA24, cleaving of this region by si5U completely inhibited the propagation of virus, thus appearance of CPE (Fig.1E), virus titer, virus copy number and level of immunofluorescence (Fig.2E and Fig.3). Cre located in 2C region is essential for genome amplification. This region is extremely sensitive to genetic alteration [24]. In addition, the Cre region spatially forms a large loop, allowing easy access to the virus replication machinery and thus to siRNA. However, satisfactory inhibition was not achieved by siCre. Polymerase $\left(3 \mathrm{D}^{\mathrm{pol}}\right)$ encodes the viral RNA-dependent RNA polymerase which organizes into a complex form and subsequently binds to the viral RNA. Since this gene and the other cellular factors are important components of viral replication, its inhibition could produce the most potent inhibitory effect on replication [25]. Targeting $3 \mathrm{D}$ by si3 $\mathrm{D}^{\mathrm{pol}}$ shown nearly same level of inhibition as obtained by si5U in cell culture (Fig.1G). Similar to 5'UTR, 3'UTR also contains secondary structural elements which consist of three stem-loops, followed by a poly (A) tail sequence. The evidence came into existence that being consisted of extensive stretches of conserved residues, 3'UTR enhances the IRES activity and IRES-mediated translation [26]. Since CA24 propagates at a good pace in cell culture system and causes prominent cytopathic effect [Fig.1B], we used a culture of it rather than the sub-genomic constructs to test the antiviral potency of siRNA. The pre-transfected Hep2 cells with si5U and si3D ${ }^{\text {pol }}$ found nearly refrained to cytopathic effect relative to infection control even after 72 hours of infection (Fig.1E and $1 G)$. IFA is a method to validate efficacy of siRNA by specific siRNA duplexes using monoclonal as primary and polyclonal as secondary antibody. The method is used to confirm the reduction of specific protein expression and the cellular immunofluorescence. In immunofluorescence assay of pre transfected Hep2 cells individually by si5U and si3 $\mathrm{D}^{\mathrm{pol}}$ followed by CA24 infection, 1-2\% fluorescence was seen relative to level of fluorescence as seen in infection control, mock and siRNA transfected cells (Fig.2).To correlate anti-CA24 potential of these siRNA, all tests and their results are shown combindly in a single plot (Fig.3). The indirect impact of virus over cells morphology (CPE and IFA) virus titer, virus copy number in si5U and si3 $\mathrm{D}^{\mathrm{pol}}$ transfected cells shown their full resistance for propagation of CA24.All experiments were performed in 3 biological and 3 technical replicates for consistency of obtained data $(p<0.05)$. The error-prone replication mechanism and high mutation rate of RNA viruses allows them to readily escape from RNAi and emergence of 
escape mutants [27]. In case of escape mutants, targeting several regions of virus at a time in the form of combination therapy of siRNA cocktail may provide better protection from virus effect. Among all four siRNA very low antiviral activity was observed in siCre and si3U. The reason may be non-accession of target sites by processed siRNA due to (I) RNA binding proteins [28], (II) Thermodynamic difference may also cause poor incorporation of siRNA to RISC [29], (III) Secondary or tertiary structures create steric hindrance resulting in difference level of positional effects [30]. The reports originated from several studies in support and against of secondary structures created a dilemma on an impact of siRNA efficiency. To check the synergistic effect of our designed siRNA, we tested them alone and in combinations but no significant difference was observed in their antiviral potency (data not shown). The reason for the failure of synergistic effect may be competition of processed siRNA for RISC by which their independent activity reduced [31].

\section{Conclusion}

In conclusion, effective siRNA-based siRNA strategy has developed against targeting 5'UTR and polymerase (3D). These siRNA are strong enough to inhibit CA24 propagation in Hep2 cells. Further, in vivo testing is required to develop siRNA-based therapeutics to combat CA24 infections.

\section{Acknowledgement}

The work was supported by Grant-in-Aid from Department of Biotechnology (Ministry of science and technology) Government of India.

\section{References}

[1] Chang $\mathrm{CH}$, Lin KH, Sheu MM. The change of etiological agents and clinical signs of epidemic viral conjunctivitis over an 18-year period in southern Taiwan. Graefe's Arch Clin Exp Ophthalmol; 2003:241:554-60.

[2] Cabrerizo M, Echevarria JE, Otero A. Molecular characterization of a Coxsackievirus A24 variant that caused an outbreak of acute haemorrhagic conjunctivitis in Spain, 2004. J Clin Virol.2008; 43(3):323-7

[3] Shukla D, Kumar A, Srivastava S, Dhole TN. Molecular identification and phylogenetic study of Coxsackievirus A24 variant isolated from an outbreak of acute haemorrhagic conjunctivitis in India in 2010. Arch Virol. 2013; 158(3):679-84.

[4] Tavares FN, Campos Rde M, Burlandy FM, Fontella R. Molecular characterization and phylogenetic study of Coxsackievirus A24v causing outbreaks of acute haemorrhagic conjunctivitis (AHC) in Brazil. PLoS One. 2011; 6(8):e23206.

[5] De W, Huanying Z, Hui L, Corina M. Phylogenetic and molecular characterization of coxsackievirusA24 variant isolates from a 2010 acute haemorrhagic conjunctivitis outbreak in Guangdong, China. Virol J. 2012; 9:41.

[6] Fonseca MC, Sarmiento L, Resik S, Pereda N, Rodríguez H, Kourí V, et al. Isolation of Coxsackievirus A24 variant from patients with haemorrhagic conjunctivitis in Cuba, 2008-2009. J Clin Virol. 2012; 53(1):77-81.

[7] Chu PY, Ke GM, Chang CH, Lin JC, Sun CY, Huang WL, Tsai YC, Ke LY, Lin KH. Molecular epidemiology of CoxsackieA type 24 variant in Taiwan, 2000-2007. J Clin Virol. 2009; 45(4):285-91.

[8] Nygårdas M, Vuorinen T, Aalto AP, Bamford DH.Inhibition of Coxsackievirus B3 and related enteroviruses by antiviral short interfering RNA pools produced using phi6 RNA-dependent RNA polymerase. J Gen Virol. 2009;90:2468-73

[9] Alhoot MA, Wang SM, Sekaran SD. RNA interference mediated inhibition of dengue virus multiplication and entry in HepG2 cells. PLoS One. 2012; 7(3):e34060.

[10] Hacke M, Björkholm P, Hellwig A, Himmels P, Ruiz de Almodóvar C, Brügger B, Wieland F, Ernst AM. Inhibition of Ebola virus glycoprotein-mediated cytotoxicity by targeting its transmembrane domain and cholesterol. Nat Commun. 2015. 9;6:7688

[11] Bivalkar-Mehla S, Mehla R, Chauhan A.Chimeric peptide-mediated siRNA transduction to inhibit HIV-1 infection. J Drug Target. 2017;25(4):307-319

[12] Braga AC, Carneiro BM, Batista MN, Akinaga MM, Rahal P. Inhibition of hepatitis C virus using siRNA targeted to the virus and Hsp90. Cell Stress Chaperones. 2017; 22(1):113-122.

[13] Zocher G, Mistry N, Frank M, Hähnlein-Schick, Ekström J, Arnberg N, Stehle T. A sialic acid binding site in a human picornavirus. PLoS Pathog. $2014 ; 10(10):$ e1004401

[14] Lin JY, Chen TC, Weng KF, Chang SC, Chen LL, Shih SR: Viral and host proteins involved in picornavirus life cycle. Biomed Sci. 2009; $16: 103$

[15] Jun EJ, Nam YR, Ahn J, et al. Antiviral potency of a siRNA targeting a conserved region of coxsackievirus A24. Biochem Biophys Res Commun. 2008; 376:389-394.

[16] Jun EJ, Won MA, Ahn J. An antiviral small-interfering RNA simultaneously effective against the most prevalent enteroviruses causing acute haemorrhagic conjunctivitis. Invest Ophthalmol Vis Sci. Jan 2011; 52(1):58-63.

[17] PHELAN, M., C., Basic Techniques for Mammalian Cell Tissue Culture. In: Current Protocols in Cell Biology, Phelan MC (ed.), New York: John Wiley \& Sons, Inc., 1998

[18] Reed L.J, Muench H. A simple method of estimating fifty percent endpoints. The American Journal of Hygiene.1938; 27 : 493-497.

[19] Chatterjee S, Quarcoopome CO, Apenteng A. An epidemic of acute conjunctivitis in Ghana. Ghana Med J.1970; 9:9-11.

[20] Yokota, T., N. Sakamoto, N. Enomoto, Y. Tanabe, M. Miyagishi. Inhibition of intracellular hepatitis C virus replication by synthetic and vector-derived small interfering RNAs.2003; EMBO Rep. 4:602-608.

[21] Jacque, J. M., K. Triques, and M. Stevenson. Modulation of HIV-1 replication by RNA interference. 2002; Nature 418:435-438.

[22] Yuan J, Cheung PK, Zhang HM, Chau D. Inhibition of coxsackievirus B3 replication by small interfering RNAs requires perfect sequence match in the central region of the viral positive strand.2005 ; J Virol.79(4):2151-9.

[23] Pan QW, Henry SD, Scholte BJ, New therapeutic opportunities for Hepatitis C based on small RNA. World J Gastroenterol. 2007; 13(33): 4431-4436.

[24] Cordey S, Gerlach D, Junier T, Zdobnov EM, Kaiser L, RNA.2008;14(8), 1568-1578

[25] Kempf BJ, Kelly MM, Springer CL, Peersen O. Structural Features of a Picornavirus Polymerase Involved in the Polyadenylation of Viral RNA. J Virol. 2013; 87(10): 5629-5644.

[26] Souii A, Gharbi J. Int. J. Mol. Sci.2013;14(3), 4525-4544 
[27] Presloid JB, Novella IS. RNA Viruses and RNAi: Quasispecies Implications for Viral Escape. Viruses. 2015; 7(6): 3226-3240.

[28] Song JJ, Smith SK, Hannon GJ, Joshua-Tor L. Crystal structure of Argonaute and its implications for RISC slicer activity. Science. 2004;305(5689):1434-7

[29] Watts JK, Corey DR, Gene silencing by siRNAs and antisense oligonucleotides in the laboratory and the clinic. J Pathol. 2012; 226(2): 365-379.

[30] Luo KQ, Chang DC. The gene-silencing efficiency of siRNA is strongly dependent on the local structure of mRNA at the targeted region. Biochem Biophys Res Commun. 2004; 318(1):303-10.

[31] Koller E, Propp S, Murray H, Lima Competition for RISC binding predicts in vitro potency of siRNA. Nucleic Acids Res. 2006; 34: 4467-4476 